\section{Therapies for depression must also be flexible to deliver}

I enjoyed David Taylor's spirited defence of the use of dynamic therapies in depression but feel that his clear model allegiance may have led to the neglect of other practical considerations (Taylor 2008).

The popular use of cognitive-behavioural therapy (CBT) in depression and other disorders is not solely due to 'a homoeopathic fantasy that brief psychological treatments are highly potent'. In comparing any National Health Service treatment, efficacy is only one consideration. Cost-effectiveness and flexibility in delivery are other issues to take into account. I believe that CBT may be superior to dynamic therapy in these regards. The recent and compelling Layard economic argument for the cost-effectiveness of CBT in adult depression has prompted the government's Improved Access to Psychological Therapies (IAPT) initiative. It is difficult to see how psychodynamic therapies could be delivered in such a responsive way, consistent with a stepped-care model. A relative advantage of CBT is that single threads of therapy at different levels of intensity can be titrated to patient need, rather than a blanket execution of the full CBT model in every case (Lovell 2000). This is supported by the finding of Jacobson et al (1996) that many patients with depression improve with behavioural activation alone. What single threads of psychodynamic therapy could be similarly utilised, independent of the complex frame that dynamic therapy usually demands?

Lovell \& Richards (2000) argue that the traditional focus on 'high intensity multiple-thread interventions' to a select few disenfranchises the remainder of people who would benefit from, but cannot access, briefer and simpler interventions. The current delivery of traditional psychodynamic therapy in traditional settings with traditionally long waiting lists is particularly vulnerable to this criticism. Whyte (1996) reports that a wider range of patients might be able to access dynamic therapy if their psychiatrists were not so deterred by the waiting lists. The coherence of CBT also lends itself more readily to training and the increased dispersion of less intensive treatments to patients directly through guided self-help materials (including computerised $\mathrm{CBT}$ ) or indirectly through the multidisciplinary team and the new models of brief training such as the SPIRIT (Structured Psychosocial InteRventions In Teams) course (Whitfield 2003).

David Taylor makes a convincing case that dynamic therapy may be as effective as CBT in depression but he does not suggest how dynamic therapy can be as coherent, cost-effective and deliverable in busy clinical settings. His argument fuels the polarised argument of CBT v. dynamic therapy and ignores more integrative therapies such as Ryle's cognitive analytic therapy. Bateman (1997) argues that 'a creative and constructive partnership between different psychotherapies needs to develop if psychotherapeutic psychiatry is to flourish'. He goes on to suggest that the greatest threat to this 'is the partisan approach of the psychotherapies themselves'.

Bateman A (1997) Borderline personality disorder and psychotherapeutic psychiatry: an integrative approach. British Journal of Psychotherapy; 13: 489-98.

Jacobson N, Dobson K, Traux P, et al (1996) A component analysis of cognitive behavioural treatment for depression. Journal of Consulting and Clinical Psychology; 64: 295-304.

Lovell K, Richards D (2000) Multiple Access Points and Levels of Entry (MAPLE): ensuring choice, accessibility and equity for CBT services. Behavioural and Cognitive Psychotherapy; 28: 379-91.

Taylor D (2008) Psychoanalytic and psychodynamic therapies for depression: the evidence base. Advances in Psychiatric Treatment; 14: 401-13.

Whitfield G, Williams C (2003) The evidence base for cognitive-behavioural therapy in depression: delivery in busy clinical settings. Advances in Psychiatric Treatment; 9: 21-30.

Whyte CR (1996) The need for dynamic psychotherapy. Psychiatric Bulletin; 20: $541-2$.

Noel D. Collins Specialist Registrar, Central and North West London Foundation Trust, Joint Homelessness Team, Soho Centre for Health and Care, 1 Frith Street, London W1D 3HZ, UK. Email: noelcollins@nhs.net doi: 10.1192/apt.15.2.159

\section{Propranolol treatment of traumatic memories}

Jonathan Bisson (2007) discusses the use of propranolol in the prevention of post-traumatic stress disorder, reported in a study by Pitman et al (2002). The latter found encouraging results with patients who had experienced very recent trauma. Brunet et al (2008) reported similar findings in patients with long-term traumatic memories (mean duration of about 10 years). These studies, as well as one by Vaiva et al (2003), recorded physiological measures such as heart rate, but did not report on any clinical benefits, for example reduced distress or changes in the integrity of the traumatic memories.

Almost a decade ago, Nader et al (2000) rekindled the reconsolidation hypothesis of memory. In brief, it proposes that when long-term memories are reactivated through retrieval, they remain labile for several hours before conversion to long-term memory. During this period they are susceptible to amnestic agents such as propranolol. It is thought that propranolol blocks the adrenaline-induced state of high arousal, which is believed to be important 
in giving traumatic memories their unique quality, including the potency and immediacy they possess (Pitman, 1989).

To explore any clinical benefits in terms of reducing distress and changes in memory integrity, I have treated six patients with a single dose of propranolol, sometimes in conjunction with a single dose of long-acting propranolol. They were asked to retrieve their traumatic memories by talking about them. Bringing these memories into immediate memory was accompanied by varying degrees of visible distress, anxiety and dissociation. They were given propranolol within $3 \mathrm{~h}$ of retrieval.

One patient with a 38-year-old memory, who had been on regular atenolol for 14 years, reported no change in the intensity, frequency, integrity or distress associated with the traumatic memory. The other five patients, with memories ranging in age from 4 months to 31 years, all reported improvement. This included 'fragmentation' of the memory and difficulty accessing it, minimal or absent distress when thinking about it and a feeling of emotional detachment, as if it were a normal non-traumatic memory or had happened to someone else. Post-treatment, several of the patients requested propranolol treatment for other traumatic memories. Benefits have been maintained for up to 4 months, with no relapse to date. None of the patients reported any negative effects from retrieving the memory, even the patient who did not respond, and side-effects from propranolol (sedation, dry mouth) were mild and transient.

These clinical cases suggest that propranolol may prove to be effective, cheap, simple and safe in the treatment of traumatic memories, which are characteristic of post-traumatic stress disorder. However, randomised controlled trials are needed to confirm any efficacy of this treatment.

Bisson JI (2007) Pharmacological treatments of post-traumatic stress disorder. Advances in Psychiatric Treatment; 13: 119-26.

Brunet A, Orr SP, Tremblay J, et al (2008) Effects of post-retrieval propranolol on psychophysiologic responding during subsequent script-driven traumatic imagery in post-traumatic stress disorder. Journal of Psychiatric Research; 42 : 503-6.

Nader K, Schafe G, Le Doux J (2000) Fear memories require protein synthesis in the amygdala for reconsolidation after retrieval. Nature; 406: 722-6.

Pitman R (1989) Post-traumatic stress disorder, hormones and memory. Biological Psychiatry; 26: 221-3

Pitman R, Sanders K, Zusman R, et al (2002) Pilot study of secondary prevention of posttraumatic stress disorder with propranolol. Biological Psychiatry; 51: 189-92.

Vaiva G, Ducrocq F, Jezequel K, et al (2003) Immediate treatment with propranolol decreases posttraumatic stress disorder two months after trauma. Biological Psychiatry; 54: 947-9.

Robin P. D. Menzies Clinical Assistant Professor, Department of Psychiatry, University of Saskatchewan, Knox Manse, 505 4th Avenue North, Saskatoon, Saskatchewan, S7K 2M5, Canada. Email: bogey@ sasktel.net

doi: 10.1192/apt.15.2.159a 